\title{
Tuberculous otitis media with facial paralysis: microbiological and clinical study
}

\author{
Adriana Mosca', Raffaele Del Prete', Nicola Quaranta' ${ }^{2}$ Luisa Miragliotta' \\ I Sezione Microbiologia, MIDIM, Università di Bari \& U.O.C. Microbiologia e Virologia \\ 2 Clinica ORL “G. Lugli”, Azienda Ospedaliera Universitaria Policlinico Bari
}

Key words: Tuberculosis, Otitis media, Swab ear secretion, Real-Time PCR

Otite media tubercolare associata con paralisi del nervo faciale: studio microbiologico e clinico

\section{SUMMARY}

Tuberculosis is a serious infectious disease affecting various organs and tissues even if the lung is the most commonly involved site. A case of tuberculous otitis media in a patient who had no history of tuberculosis is herewith presented with the aim of increasing the awareness of this disease whose diagnosis is often delayed because either of the rarity of this pathologic condition or of its usually indolent course.

\section{INTRODUZIONE}

La tubercolosi è una malattia infettiva altamente contagiosa che può interessare vari organi ed apparati anche se il polmone è il sito più comunemente coinvolto nella forma primaria. Le più comuni localizzazioni extrapolmonari sono pleura, linfonodi, sistema nervoso centrale, cute, intestino, ghiandole surrenali, apparato visivo e laringe (3). L'otite media tubercolare (TOM) è invece rara e spesso di non facile diagnosi. Essa è di norma secondaria alla patologia polmonare o in organi adiacenti, quali laringe, faringe e naso, mentre come forma primaria è un evento estremamente raro (2).

Si descrive qui il caso di una paziente con tubercolosi primaria dell'orecchio medio e del mastoide destro senza alcuna evidenza di infezione tubercolare in altri distretti.

Lo scopo di tale studio è quello di ottimizzare aumentare la consapevolezza di tale possibilità e di accelerare la diagnosi spesso ritardata a causa della rarità della patologia o del decorso indolore $(6,5)$.

\section{CASO CLINICO}

La paziente di sesso femminile, 87 anni, era affetta da fibrosi polmonare, broncopolmonite cronica ostruttiva, insufficienza cardiaca cronica, ed era stata ricoverata in clinica otoiatrica, Policlinico di Bari, per otorrea, otalgia e paralisi facciale del lato destro. Nel 2009 la paziente era stata sottoposta a meatoplastica. Esami ematochimici ed esami radiografici del torace erano nella norma.

La TAC del temporale di destra evidenziava presenza di tessuto infiammatorio nel meato uditivo esterno che all'esame istologico evidenziava flogosi granulomatosa di tipo tubercolare con discre- ta necrosi caseosa (Figura I).

L'esame batterioscopico, eseguito su materiale prelevato mediante tampone auricolare, è risultato negativo alla ricerca di bacilli acido-alcool resistenti (BAAR).

Il materiale sottoposto ad esame colturale con il sistema BACTEC MGIT 960 (Becton Dickinson, Italia) ha dato esito positivo dopo 20 giorni.

L'identificazione è stata effettuata con metodo molecolare: previa estrazione degli acidi nucleici con il sistema EZ1 Robot (QIAGEN, Germania) e Real-Time PCR per la ricerca di DNA di Mycobacterium tuberculosis complex (Artus M. tuberculosis RG PCR, QIAGEN, Germania).

Tale metodica ha dato esito positivo permettendo di porre la diagnosi di infezione tubercolare.

La terapia antitubercolare, tempestivamente somministrata, ha risolto la sintomatologia a carico dell'orecchio con regressione della paralisi del nervo facciale.

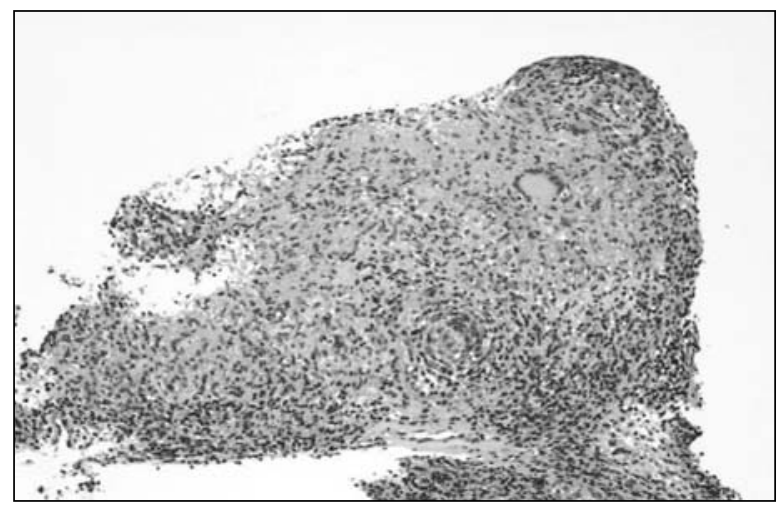

Figura I. Infiltrato infiammatorio con aspetto granulomatoso, cellule epitelioidi e cellule giganti plurinucleate tipo Langhans (100x). 


\section{CONCLUSIONI}

La diagnosi di otite media tubercolare è spesso ritardata sia per la sua bassa incidenza $(0.05-0.9 \%)$ (5) sia per la presenza di sintomi non dissimili da quelli dell'otite media di altra eziologia (1).

$\mathrm{Nel}$ nostro caso inoltre le manifestazioni cliniche non erano perfettamente sovrapponibili alla descrizione classica che prevede la triade: otorrea, paralisi del nervo facciale e perforazioni multiple del timpano (6). In letteratura, fra l'altro, il maggior numero di casi di TOM riguardano bambini $(15-40 \%)$ mentre il nostro caso considera una paziente anziana (5).

In base alle attuali conoscenze il caso di TOM qui riportato risulta essere il primo dovuto ad infezione primaria in seguito a meatoplastica.

Il tipico quadro tubercolare dell'esame istologico è stato confermato dalle indagini microbiologiche, anche se la colorazione Ziehl Neelsen è risultata negativa verisimilmente a causa dell'impiego, per il prelievo, di un tampone che certamente non è il metodo ottimale per la raccolta di materiali purulenti. Le tecniche molecolari, che permettono di avere risultati con elevata specificità e sensibilità in poche ore, sono indispensabili per una terapia più immediata ed efficace evitando così possibili complicanze.
Il caso presentato conferma l'utilità del ricorso alla diagnostica molecolare della tubercolosi anche su materiali purulenti. E ciò nonostante che la maggioranza dei kit di amplificazione vengano commercializzati solo per i materiali di origine polmonare e nonostante lo scetticismo delle linee guida dei Centers of Disease Control and Prevention (7).

\section{BIBLIOGRAFIA}

1. Awan MS, Salahuddin I. Tuberculous otitis media: two case reports and literature review. Ear Nose Throat J 2002; 81: 792-4.

2. Davidson S, Creter D, Leventon G, Katznelson D. Tuberculosis of the middle ear in an infant. Arch Otolaryngol Head Neck Surg 1989; 115: 876-7.

3. Frieden TR, Munsiff SS, Watt CJ, Dye C. Tuberculosis. Lancet 2003; 362: 887-99.

4. Lee PY, Drysdale AJ. Tuberculous otitis media: a difficult diagnosis. J Laryngol Otol 1993; 107: 339-41.

5. Vaamonde P, Castro C, Garcì-Soto N, Labella T, Lozano A. Tuberculous otitis media: a significant diagnostic challenge. Otolatyngol Head Neck Surg 2004; 130: 759-66.

6. Vital V, Printza A, Zaraboukas T. Tuberculous otitis media: a different diagnosis and report of four cases. Pathol Res Pract 2002; 198: 31-5.

7. C.D.C. Updated guidelines for the use of nucleic acid amplification tests in the diagnosis of tuberculosis. MMWR 2009; 58: 7-10. 